\title{
Changes in SICAM-1 and GM-CSF levels in skin tissue fluid and expression of IL-6, IL-17 and TNF- $\alpha$ in blood of patients with vitiligo
}

\author{
XUESONG YANG, LINGYI YAN, DA HA, LINA QU, LING LIU and YU TAO \\ Department of Dermatology, Daqing Long Nan Hospital, Daqing, Heilongjiang 163453, P.R. China
}

Received April 19, 2018; Accepted October 25, 2018

DOI: 10.3892/etm.2018.6937

\begin{abstract}
Changes in the levels of soluble intercellular adhesion molecule-1 (sICAM-1) and granulocyte-macrophage colony stimulating factor (GM-CSF) in the skin tissue fluid, and the expression of interleukin (IL)-6, IL-17 and tumor necrosis factor- $\alpha(\mathrm{TNF}-\alpha)$ in the blood of patients with vitiligo were investigated. One hundred and twenty patients diagnosed with vitiligo and treated in Daqing Long Nan Hospital from March 2014 to March 2016 were selected, including 88 patients with vitiligo vulgaris and 32 patients with segmental vitiligo. Comparative analyses were performed for research indexes. Another 80 healthy volunteers receiving physical examination were selected as healthy controls. The levels of GM-CSF in tissue fluid were detected via radioimmunoassay (RIA). The levels of SICAM-1 in tissue fluid and IL-6, IL-17 and TNF- $\alpha$ in the blood were detected via enzyme-linked immunosorbent assay (ELISA). The expression levels of IL-6, IL-17 and TNF- $\alpha$ in patients with progressive vitiligo were significantly higher than those in patients with stable vitiligo $(\mathrm{P}<0.05)$. The levels of sICAM-1 and GM-CSF in the skin tissue fluid at white spots of patients with vitiligo vulgaris were significantly higher than those in the skin tissue fluid at non-white spots $(\mathrm{P}<0.05)$. sICAM-1 levels had significant positive correlations with the levels of IL-6, IL-17 and TNF- $\alpha$ in the blood $(\mathrm{P}<0.05)$. The levels of sICAM-1 in the skin tissue fluid and IL-6 in the blood of patients with vitiligo were negatively correlated with the course of disease $(\mathrm{P}<0.05)$. The levels of sICAM-1 in the skin tissue fluid and IL-6 and IL-17 in the blood of patients with vitiligo were positively correlated with the skin lesion area of patients $(\mathrm{P}<0.05)$. The levels of sICAM-1 and GM-CSF in the skin tissue fluid, and the expression levels of IL-6, IL-17 and TNF- $\alpha$ in the blood of patients with vitiligo are abnormal.
\end{abstract}

Correspondence to: $\mathrm{Dr} \mathrm{Yu}$ Tao, Department of Dermatology, Daqing Long Nan Hospital, 35 Aiguo Road, Daqing, Heilongjiang 163453, P.R. China

E-mail: sptsxy1@163.com

Key words: vitiligo, sICAM-1, GM-CSF, IL-6, IL-17, TNF- $\alpha$

\section{Introduction}

Vitiligo is a dermatogic disease (1), whose pathogenesis is currently only slightly understood. Vitiligo is a melanocyte-specific damage disease, and many scholars have speculated that the occurrence of vitiligo is very likely to be caused by the autoimmune dysfunction of patients $(2,3)$. There is a variety of cytokines in the body which can form a very complex immune regulatory network through the mutual feedback and regulatory effect of synthesis and secretion, and the mutual influence, regulatory effect and biological effect of the expression of cytokine receptors $(4,5)$. Abdellatif et al $(6)$ have proposed that the proliferation activity of $\mathrm{T}$ cells in the peripheral blood of patients with vitiligo is abnormal, and the $\mathrm{T}$ cell-mediated normal melanocyte antigen response plays an important role in the occurrence of vitiligo. In the present study, in order to further investigate the pathogenesis of vitiligo, the levels of soluble intercellular adhesion molecule-1 (sICAM-1) and granulocyte-macrophage colony stimulating factor (GM-CSF) in the skin tissue fluid and the levels of interleukin (IL)-6, IL-17 and tumor necrosis factor- $\alpha$ (TNF- $\alpha$ ) in the blood of patients with vitiligo were detected, and their correlations with the course of disease and skin lesion area of patients were explored.

\section{Materials and methods}

General data. A total of 120 patients diagnosed with vitiligo and treated in Daqing Long Nan Hospital (Daqing, China) from March 2014 to March 2016 were selected, according to the diagnostic and staging criteria of vitiligo $(7,8)$, including 66 male patients and 54 female patients, 15-72 years of age, with the course of disease being 3 months to 15 years. Among them, 60 patients of 14-70 years of age, with the course of disease being 2 months to 14 years, were in the progressive stage; and 60 patients of 16-72 years of age, with the course of disease being 4 months to 16 years, were in the stable stage. The skin lesion areas of all patients enrolled were recorded and presented as percentage. Another 80 healthy volunteers receiving physical examination in Daqing Long Nan Hospital during the same period were selected as control group, including 44 males and 36 females, 18-70 years of age. The age and sex of all subjects in vitiligo and control group were comparable $(\mathrm{P}>0.05)$. 
Table I. Expression of IL-6, IL-17 and TNF- $\alpha$ in the blood of patients of both groups (mean \pm standard deviation).

\begin{tabular}{lcccc}
\hline Group & $\mathrm{n}$ & $\mathrm{IL}-6(\mathrm{ng} / \mathrm{l})$ & $\mathrm{IL}-17(\mathrm{~g} / \mathrm{l})$ & $\mathrm{TNF}-\alpha(\mu \mathrm{g} / \mathrm{l})$ \\
\hline $\begin{array}{l}\text { Healthy } \\
\text { control }\end{array}$ & 80 & $104.29 \pm 37.61$ & $9.74 \pm 3.48$ & $0.88 \pm 0.25$ \\
Vitiligo & 120 & $188.47 \pm 58.42^{\mathrm{a}}$ & $14.29 \pm 4.31^{\mathrm{a}}$ & $2.04 \pm 0.73^{\mathrm{a}}$ \\
\hline
\end{tabular}

${ }^{\mathrm{a}} \mathrm{P}<0.05$ vs. healthy control group. IL, interleukin; TNF- $\alpha$, tumor necrosis factor- $\alpha$.

Table II. Expression of IL-6, IL-17 and TNF- $\alpha$ in the blood of patients with vitiligo at different stages (mean \pm standard deviation).

\begin{tabular}{lcccc}
\hline Group & $\mathrm{n}$ & $\mathrm{IL}-6(\mathrm{ng} / \mathrm{l})$ & $\mathrm{IL}-17(\mathrm{~g} / \mathrm{l})$ & $\mathrm{TNF}-\alpha(\mu \mathrm{g} / \mathrm{l})$ \\
\hline $\begin{array}{l}\text { Healthy } \\
\text { controls }\end{array}$ & 80 & $104.29 \pm 37.61^{\mathrm{a}}$ & $9.74 \pm 3.48^{\mathrm{a}}$ & $0.88 \pm 0.25^{\mathrm{a}}$ \\
$\begin{array}{l}\text { Patients in } \\
\text { progressive stage }\end{array}$ & 60 & $238.42 \pm 55.29$ & $17.46 \pm 4.99$ & $2.52 \pm 0.96$ \\
$\begin{array}{l}\text { Patients in } \\
\text { stable stage }\end{array}$ & 60 & $112.49 \pm 40.05^{\mathrm{a}}$ & $10.61 \pm 4.16^{\mathrm{a}}$ & $1.16 \pm 0.44^{\mathrm{a}}$ \\
\hline
\end{tabular}

${ }^{a} \mathrm{P}<0.05$ vs. patients in the progressive stage. IL, interleukin; TNF- $\alpha$, tumor necrosis factor- $\alpha$.

Exclusion criteria: patients with severe cardiovascular or cerebrovascular diseases, liver or kidney disease, infection, tumor or other autoimmune diseases, or patients receiving any immunotherapy within 1 month before detection.

The study was approved by the Ethics Committee of Daqing Long Nan Hospital and informed consents were signed by the patients or the guardians.

\section{Methods}

Collection of skin tissue fluid. The blebs at white and non-white spots of patients with vitiligo were sucked via negative pressure suction using a negative pressure suction instrument (Jiangsu Keling Medical Appliances Co., Ltd., Jiangsu, China). More visible blebs could be seen after $2 \mathrm{~h}$. The bleb fluid at white and non-white spots was collected using a sterile disposable syringe, and it was stored in a refrigerator at $-20^{\circ} \mathrm{C}$ to be detected.

Collection of serum. A total of $5 \mathrm{ml}$ fasting blood was collected into anticoagulant tubes from vitiligo patients and healthy volunteers receiving physical examination in the morning. The samples were placed in the refrigerator at $-4^{\circ} \mathrm{C}$ for $2 \mathrm{~h}$. The serum of the two groups was collected from the blood via centrifugation at $1,300 \mathrm{xg}$ for $5 \mathrm{~min}$ at $4^{\circ} \mathrm{C}$, labeled and placed in the refrigerator at $-20^{\circ} \mathrm{C}$ in order to be detected.

Detection of SICAM-1 levels in the skin tissue fluid via radioimmunoassay (RIA) kit. The RIA kit was purchased from Shanghai Kemin Biotechnology Co., Ltd. (Shanghai, China). DFM-96 RIA $\gamma$ counter was used as the measuring instrument and the detection was performed strictly according to the manufacturer's instructions of the RIA kit.
Table III. Levels of sICAM-1 and GM-CSF in the skin tissue fluid of patients with vitiligo (mean \pm standard deviation).

\begin{tabular}{lccc}
\hline Group & $\mathrm{n}$ & $\begin{array}{c}\text { sICAM-1 } \\
(\mathrm{ng} / \mathrm{ml})\end{array}$ & $\begin{array}{c}\text { GM-CSF } \\
(\mathrm{ng} / \mathrm{ml})\end{array}$ \\
\hline Vitiligo vulgaris & 88 & & \\
White spots & 44 & $285.42 \pm 39.71$ & $0.57 \pm 0.08$ \\
$\quad$ Non-white spots & 44 & $164.27 \pm 31.38^{\mathrm{a}}$ & $0.24 \pm 0.05^{\mathrm{a}}$ \\
Segmental vitiligo & 32 & & \\
White spots & 16 & $151.49 \pm 41.36$ & $0.51 \pm 0.07$ \\
Non-white spots & 16 & $147.52 \pm 46.03^{\mathrm{b}}$ & $0.48 \pm 0.06^{\mathrm{b}}$ \\
\hline
\end{tabular}

${ }^{\mathrm{a}} \mathrm{P}<0.05,{ }^{\mathrm{b}} \mathrm{P}>0.05$ vs. white spots. sICAM-1, soluble intercellular adhesion molecule-1; GM-CSF, granulocyte-macrophage colony stimulating factor.

Detection of serum IL-6, IL-17, TNF- $\alpha$ and GM-CSF levels via enzyme-linked immunosorbent assay (ELISA) kit. IL-6, IL-17 and TNF- $\alpha$ ELISA kits were purchased from Shanghai Jingkang Biological Engineering Co., Ltd. (Shanghai, China), and the GM-CSF ELISA kit was purchased from Shanghai Beinuo Biotech Co., Ltd. (Shanghai, China). Bio-Rad 550 full-automatic enzyme-labeling counter (Bio-Rad Laboratories, Inc., Hercules, CA, USA) was used as the measuring instrument, and the detection was performed strictly according to the manufacturer's instructions of the ELISA kit.

Statistical analysis. Statistical Product and Service Solutions (SPSS) 19.0 software (IBM Corp., Armonk, NY, USA) was used for the statistical analysis of the data. Measurement data are presented as mean \pm standard deviation. t-test was used for the intergroup comparison. ANOVA was used for comparison between multiple groups and the post hoc test was LSD test. The Pearson's correlation test was utilized to analyze correlation. $\alpha=0.05$ was used as the inspection level.

\section{Results}

Expression of IL-6, IL-17 and TNF- $\alpha$ in the blood of patients of both groups. The expression levels of IL-6, IL-17 and TNF- $\alpha$ in the blood of patients in the healthy control group were significantly lower than those in the vitiligo group, and the differences were statistically significant $(\mathrm{P}<0.05)$ (Table I).

IL-6, IL-17 and TNF- $\alpha$ in the blood of patients with vitiligo in different stages. There were no significant differences in the expression levels of IL-6, IL-17 and TNF- $\alpha$ in the blood of patients of the healthy control group and the patients at the stable stage; but the levels were significantly lower than those in the blood of patients in progressive stage, and the differences were statistically significant $(\mathrm{P}<0.05)$ (Table II).

Levels of sICAM-1 and GM-CSF in the skin tissue fluid of patients with vitiligo. The levels of SICAM-1 and GM-CSF in the skin tissue fluid at white spots of patients with vitiligo vulgaris were significantly higher than those in the skin tissue 

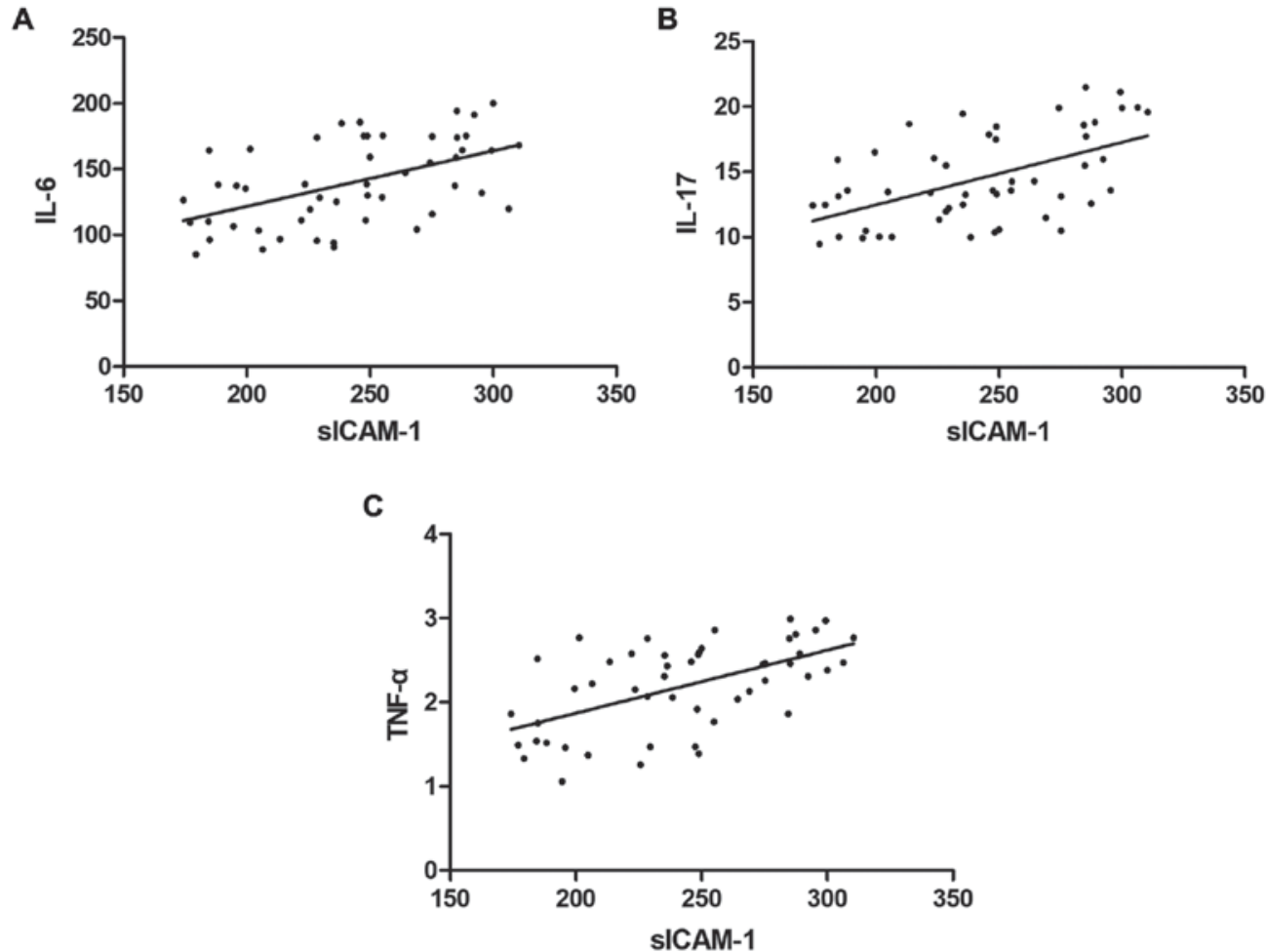

Figure 1. Correlation of sICAM-1 levels in the skin tissue fluid with the (A) IL-6, (B) IL-17 and (C) TNF- $\alpha$ levels in the blood. sICAM-1, soluble intercellular adhesion molecule-1; IL, interleukin; TNF- $\alpha$, tumor necrosis factor- $\alpha$.

A

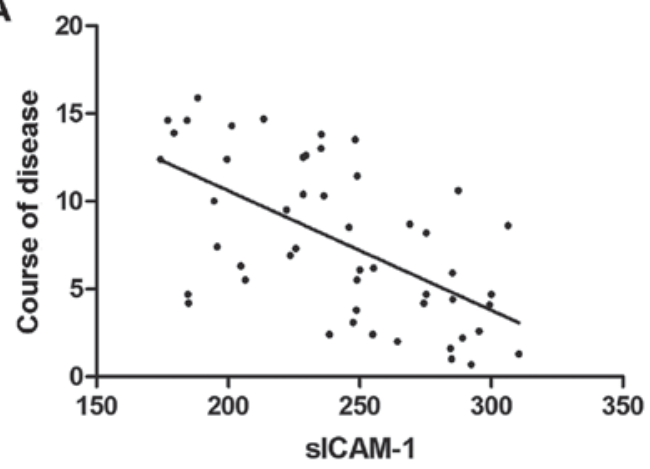

B

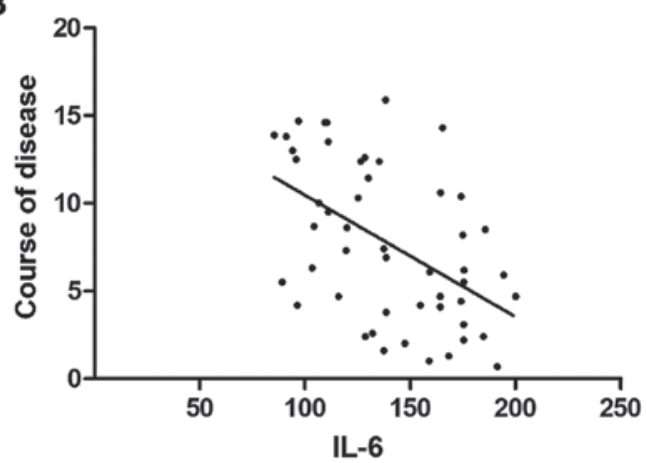

Figure 2. Correlation of (A) sICAM-1 and (B) IL-6 levels with the course of disease. sICAM-1, soluble intercellular adhesion molecule-1; IL, interleukin.

fluid at non-white spots, and the differences were statistically significant $(\mathrm{P}<0.05)$. There were no significant changes in the levels of SICAM-1 and GM-CSF in the skin tissue fluid at white and non-white spots of patients with segmental vitiligo, and the differences were not statistically significant $(\mathrm{P}>0.05)$ (Table III).

Correlation of SICAM-1 levels in the skin tissue fluid with the levels of IL-6,IL-17 and TNF- $\alpha$ in the blood. Pearson's correlation test showed that the sICAM-1 levels in the skin tissue fluid were significantly positive correlated with the levels of IL-6, IL-17 and TNF- $\alpha$ in the blood (correlation coefficient $\mathrm{r}=0.5141,0.5423$ and $0.5657, \mathrm{P}<0.05)$ (Fig. 1).

Correlation of SICAM-1 and IL-6 levels with the course of disease of patients. Pearson's correlation test showed that the levels of sICAM-1 in the skin tissue fluid and IL-6 in the blood of patients with vitiligo were negatively correlated with the course of disease $(r=-0.5999$ and $-0.5013, \mathrm{P}<0.05$ and $\mathrm{P}=0.0002)$ (Fig. 2).

Correlation of the factor levels with the skin lesion area of patients. Pearson's correlation test showed that the levels of sICAM-1 in the skin tissue fluid and IL-6 and IL-17 in the blood of patients with vitiligo were positively correlated with the skin lesion area of patients $(r=0.3772,0.4353$ and 0.3668 , $\mathrm{P}=0.0069,0.0016$ and 0.0088 ) (Fig. 3).

\section{Discussion}

sICAM-1 is a shedding cycle mode of ICAM-1 which mainly mediates the inflammatory response among cells, as a kind of adhesion molecule (9). Aydıngöz et al (10) have found that there is a significant positive correlation between ICAM-1 and sICAM-1. Camara-Lemarroy and Salas-Alanis (11) have shown that the expression level of SICAM-1 in the blood of patients 

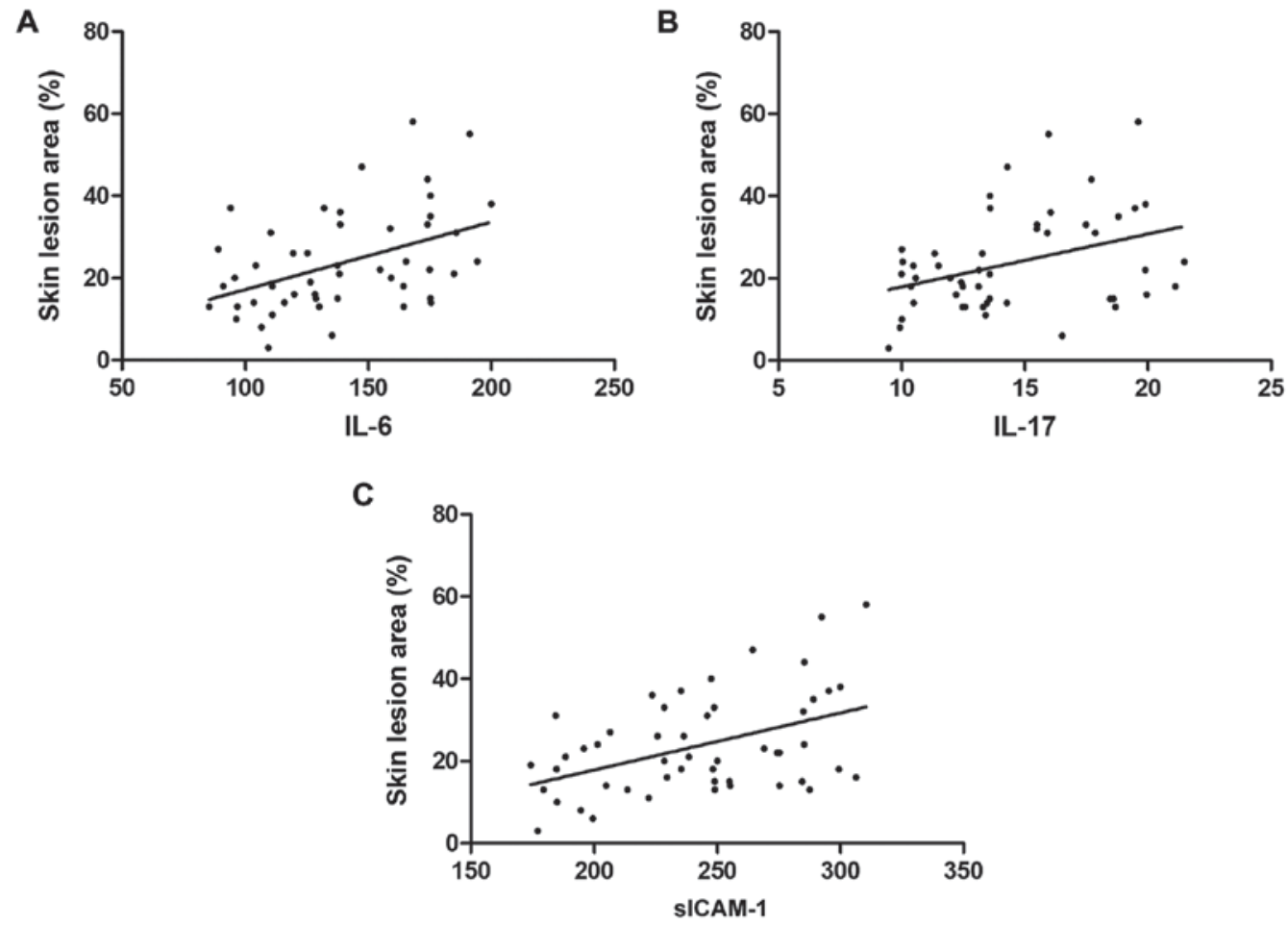

Figure 3. Correlation of (A) IL-6, (B) IL-17 and (C) sICAM-1 levels with the skin lesion area of the patients. sICAM-1, soluble intercellular adhesion molecule-1; IL, interleukin.

with progressive vitiligo is higher, and it is speculated that sICAM-1 can be used as one of the indexes of vitiligo activity. Farhan et al (12) have found that lymphocyte relocation can be seen in the skin lesion, and this relocation process is synchronized with the destruction of melanocytes. Further studies have shown that ICAM-1 can promote the adhesion process in vivo, leading to the destruction of melanocytes. IL-17 in vivo can effectively promote the synthesis of interferon and TNF, further inducing the ICAM-1 expression. In this study, the levels of sICAM-1 in the skin tissue fluid at white spots of patients with vitiligo vulgaris were found to be significantly higher than that in the skin tissue fluid at non-white spots, but there were no significant changes in the levels of sICAM-1 in the skin tissue fluid at white and non-white spots of patients with segmental vitiligo, suggesting that depigmentation of skin in patients with vitiligo vulgaris is correlated with sICAM-1. The levels of sICAM-1 showed significant positive correlations with the levels of IL-6, IL-17 and TNF- $\alpha$, so it is speculated that with the increase of sICAM-1 level in the tissue fluid, the secretion of IL-6, IL-17 and TNF- $\alpha$ is promoted via cell adhesion and receptor binding, eventually leading to increased expression levels in the blood.

GM-CSF, as the mitogen of melanocytes, can provide endogenous promoters for the growth of melanocytes $(13,14)$. Zhou et al (15) have shown via animal experiments that GM-CSF can induce autoimmune gastritis in rats. However, there have been no reports on its correlation with human vitiligo so far. In this study, the levels of GM-CSF in the skin tissue fluid at white spots of patients with vitiligo vulgaris were found to be significantly higher than that in the skin tissue fluid at non-white spots, but there were no significant changes in the levels of GM-CSF in the skin tissue fluid at white and non-white spots of patients with segmental vitiligo, and the levels of GM-CSF had no significant correlations with other factors selected in this study.

IL-6, as a kind of stimulating factor, shows a variety of biological functions in vivo (16). Miniati et al (17) have found that the expression of serum IL-6 in patients with vitiligo is significantly higher than that in normal subjects. It is speculated that the increase of IL-6 levels may be due to the destruction of cellular network in patients with vitiligo. When cells secrete IL-6 continuously, IL-6 can bind to the receptor and further stimulate the proliferation of cells, thereby leading to local skin damage. It was also found in this study that the expression of serum IL-6 in patients with vitiligo is increased, the expression level of sICAM-1 is significantly related to IL-6, and the larger the skin lesion area of patients is, the higher the expression of IL- 6 will be. Moreover, the longer the course of disease is, the lower the level of IL-6 will be, presumably because the disease gradually becomes stable, and the IL- 6 level is decreased with the extension of the course of disease.

IL-17 is mainly produced by T cells (18). In this study, it was found that the expression of serum IL-17 in patients with vitiligo is increased, and it is positively correlated with the skin lesion area. Some scholars speculate that IL-17 in the body may change the local microenvironment of patients, leading to melanocyte damage and vitiligo. TNF- $\alpha$ is a kind of cytokine with dual effects, which can be involved in the immune damage of the body $(19,20)$. It was found in this study that the level of serum TNF- $\alpha$ in patients with vitiligo is increased, presumably because TNF- $\alpha$ causes immune suppression in the body, so lymphocytes cannot exert normal immune response.

In conclusion, the levels of sICAM-1 and GM-CSF in the skin tissue fluid, and the expression of IL-6, IL-17 and TNF- $\alpha$ 
in the blood of patients with vitiligo are abnormal, but there are no significant correlations of GM-CSF with the expression of other factors, and the expression of sICAM-1 and IL-6 are correlated with the course of disease and skin lesion area of patients.

\section{Acknowledgements}

Not applicable.

\section{Funding}

No funding was received.

\section{Availability of data and materials}

The datasets used and/or analyzed during the present study are available from the corresponding author on reasonable request.

\section{Authors' contributions}

XY wrote the manuscript and collected the skin tissue fluid. LY collected the serum. DH and LQ detected the sICAM-1 levels. LL and YT performed ELISA. All authors read and approved the final manuscript.

\section{Ethics approval and consent to participate}

The study was approved by the Ethics Committee of Daqing Long Nan Hospital (Daqing, China) and informed consents were signed by the patients or the guardians.

\section{Patient consent for publication}

Not applicable.

\section{Competing interests}

The authors declare that they have no competing interests.

\section{References}

1. Baldini E, Odorisio T, Sorrenti S, Catania A, Tartaglia F, Carbotta G,Pironi D, Rendina R,D'Armiento E,Persechino S, et al: Vitiligo and autoimmune thyroid disorders. Front Endocrinol (Lausanne) 8: 290, 2017.

2. Ezzedine K and Eleftheriadou V: Vitiligo and quality of life: The dark face of whiteness. Br J Dermatol 178: 28-29, 2018.

3. Dwivedi M, Laddha NC, Shah K, Shah BJ and Begum R: Involvement of interferon-gamma genetic variants and intercellular adhesion molecule-1 in onset and progression of generalized vitiligo. J Interferon Cytokine Res 33: 646-659, 2013.

4. Wu XG, Hong WS and Xu A: GM-CSF: a possible prognostic serum biomarker of vitiligo patients' considered for transplantation treatment with cultured autologous melanocytes: A pilot study. J Eur Acad Dermatol Venereol 30: 1409-1411, 2016.
5. Mitra S, De Sarkar S, Pradhan A, Pati AK, Pradhan R, Mondal D, Sen S, Ghosh A, Chatterjee S and Chatterjee M: Levels of oxidative damage and proinflammatory cytokines are enhanced in patients with active vitiligo. Free Radic Res 51: 986-994, 2017.

6. Abdellatif AA, Zaki AM, Abdo HM, Aly DG, Emara TA, El-Toukhy S, Emam HM and Abdelwahab MS: Assessment of serum levels of granulocyte-macrophage colony-stimulating factor (GM-CSF) among non-segmental vitiligo patients: A pilot study. Acta Dermatovenerol Alp Pannonica Adriat 24: 43-45, 2015.

7. Laddha NC, Dwivedi M, Gani AR, Mansuri MS and Begum R: Tumor necrosis factor B (TNFB) genetic variants and its increased expression are associated with vitiligo susceptibility. PLoS One 8: e81736, 2013.

8. Kemp EH: Tumour necrosis factor- $\alpha$ antagonists as therapies for vitiligo. Br J Dermatol 173: 635, 2015.

9. Webb KC, Tung R, Winterfield LS, Gottlieb AB, Eby JM, Henning SW and Le Poole IC: Tumour necrosis factor- $\alpha$ inhibition can stabilize disease in progressive vitiligo. $\mathrm{Br} \mathrm{J}$ Dermatol 173: 641-650, 2015.

10. Aydıngöz IE, Kanmaz-Özer M, Gedikbaşi A, Vural P, Doğru-Abbasoğlu S and Uysal M: The combination of tumour necrosis factor- $\alpha-308 \mathrm{~A}$ and interleukin-10 $-1082 \mathrm{G}$ gene polymorphisms and increased serum levels of related cytokines: Susceptibility to vitiligo. Clin Exp Dermatol 40: 71-77, 2015.

11. Camara-Lemarroy CR and Salas-Alanis JC: The role of tumor necrosis factor- $\alpha$ in the pathogenesis of vitiligo. Am J Clin Dermatol 14: 343-350, 2013.

12. Farhan J, Al-Shobaili HA, Zafar U, Al Salloom A, Meki AR and Rasheed Z: Interleukin-6: A possible inflammatory link between vitiligo and type 1 diabetes. Br J Biomed Sci 71: 151-157, 2014.

13. Manga P, Elbuluk N and Orlow SJ: Recent advances in understanding vitiligo. F1000Res 5: 2234, 2016.

14. Bhardwaj S, Rani S, Srivastava N, Kumar R and Parsad D: Increased systemic and epidermal levels of IL-17A and IL-1 $\beta$ promotes progression of non-segmental vitiligo. Cytokine 91: 153-161, 2017.

15. Zhou L, Shi YL, Li K, Hamzavi I, Gao TW, Huggins RH, Lim HW and Mi QS: Increased circulating Th17 cells and elevated serum levels of TGF-beta and IL-21 are correlated with human non-segmental vitiligo development. Pigment Cell Melanoma Res 28: 324-329, 2015.

16. Speeckaert R, Lambert J, Grine L, Van Gele M, De Schepper S and van Geel N: The many faces of interleukin-17 in inflammatory skin diseases. Br J Dermatol 175: 892-901, 2016.

17. Miniati A, Weng Z, Zhang B, Therianou A, Vasiadi M, Nicolaidou E, Stratigos AJ, Antoniou C and Theoharides TC: Stimulated human melanocytes express and release interleukin-8, which is inhibited by luteolin: Relevance to early vitiligo. Clin Exp Dermatol 39: 54-57, 2014.

18. Elela MA, Hegazy RA, Fawzy MM, Rashed LA and Rasheed H: Interleukin 17, interleukin 22 and FoxP3 expression in tissue and serum of non-segmental vitiligo: A case- controlled study on eighty-four patients. Eur J Dermatol 23: 350-355, 2013.

19. Toussirot É and Aubin F: Paradoxical reactions under TNF- $\alpha$ blocking agents and other biological agents given for chronic immune-mediated diseases: An analytical and comprehensive overview. RMD Open 2: e000239, 2016.

20. Meurer M and Ceric-Dehdari P: Systemic treatment of vitiligo: Balance and current developments. Hautarzt 68: 876-884, 2017 (In German).

This work is licensed under a Creative Commons Attribution-NonCommercial-NoDerivatives 4.0 International (CC BY-NC-ND 4.0) License. 